\title{
Identification of Differentially Expressed Proteins in the Serum for Systemic Juvenile Idiopathic Arthritis Using Next-generation Proteomics
}

Hironori Sato

Chiba University

Yuzaburo Inoue ( $\nabla$ yuzaburo@chiba-u.jp )

Chiba Children's Hospital

Yusuke Kawashima

Kazusa DNA Research Institute

Daisuke Nakajima

Kazusa DNA Research Institute

\section{Ren Nakamura}

Kazusa DNA Research Institute

Daigo Kato

Chiba Children's Hospital

Kanako Mitsunaga

Chiba Children's Hospital

Takeshi Yamamoto

Benaroya Research Institute at Virginia Mason

Akiko Yamaide

Chiba Children's Hospital

Minako Tomiita

Shimoshizu National Hospital

Akira Hoshioka

Chiba Children's Hospital

Osamu Ohara

Kazusa DNA Research Institute

Naoki Shimojo

Chiba University

\section{Research Article}

Keywords: Systemic juvenile idiopathic arthritis (sJIA), macrophage activation syndrome (MAS), Heme oxygenase 
Posted Date: February 18th, 2021

DOl: https://doi.org/10.21203/rs.3.rs-196053/v1

License: (c) (i) This work is licensed under a Creative Commons Attribution 4.0 International License. Read Full License 


\section{Abstract}

Systemic juvenile idiopathic arthritis (sJIA) is an autoinflammatory disease caused by high production of inflammatory cytokines. Conventional biomarkers, such as IL-18 are reportedly not always associated with frequent relapses or complication with macrophage activation syndrome (MAS). As few specific biomarkers that can indicate and evaluate the SJIA disease activity have been identified, the discovery of biomarkers is very important. We performed a deep proteomic analysis of serum samples from nine patients with SJIA using highly sensitive mass spectrometry, and identified differentially expressed proteins in various disease phases. We selected 68 proteins (URPs) that were highly expressed in the active phase from total of 2,727 proteins. Pathway analysis revealed that the URPs included proteins related to many immune process and proteasome proteins, which might be associated with the pathogenesis of sJIA. Based on these results, four proteins (leucine aminopeptidase 3; LAP3, guanylatebinding protein 1; GBP1, Heme oxygenase 1; HMOX1, and bone morphogenetic protein 10; BMP10), which exhibit high fold changes during the active phase or which might be core proteins in the functional network were selected as candidate biomarkers. These proteins may be clinically useful for diagnostic and therapeutic purposes and might help determine the pathogenesis of the disease.

\section{Introduction}

Systemic juvenile idiopathic arthritis (sJIA) is a chronic arthritis that causes systemic symptoms, such as a spiking high fever, salmon-pink rash, lymphadenopathy, hepatosplenomegaly, and serositis. While sJIA is considered an autoinflammatory disease with no genetic involvement(1-3), the pathogenesis leading to systemic inflammation remains unclear.

One of the clinical problems with SJIA is the difficulty diagnosing the disease at the onset because of the many differential diagnoses; biomarkers to facilitate the diagnosis are therefore needed. In addition, SJIA is sometimes complicated by macrophage activation syndrome (MAS) due to the cytokine storm induced by the activation of T lymphocytes and macrophages(2). As MAS causes rapid progression of lifethreatening conditions and requires accurate and prompt evaluations and treatment $(4,5)$, indicators for predicting MAS are also required.

Several acute phase proteins and pro-inflammatory cytokines have been used to assess the sJIA disease activity and the risk of complications with MAS. C-reactive protein (CRP) and ferritin can be measured rapidly and easily in clinical practice $(6,7)$, but these proteins are non-specific, and it takes time for them to be secreted into the blood and increase in concentration. In addition, the use of some biologics, such as tocilizumab, suppresses their production. Interleukin (IL) -18 and soluble CD163 are useful biomarkers for distinguishing SJIA from other diseases because of their rapid rise during the acute phase of SJIA and MAS(8-11). However, accurately evaluating these biomarkers can be difficult, as they cannot be measured immediately in general practice and because some sJIA patients have persistent IL-18 production despite a low disease activity(12). 
Proteome analyses using mass spectrometry (MS) have recently attracted attention as a powerful tool for elucidating the pathology and identifying disease-related proteins as candidate biomarkers. Proteome analyses can identify many proteins from micro samples(13), so applying this technique with systemic acute inflammatory diseases may reveal not only acute-phase proteins and proinflammatory cytokines located downstream of inflammatory pathways but also unknown upstream proteins that cause or enhance inflammation. Furthermore, functional and pathway analyses of the identified proteins may reveal new pathological conditions that have not been previously reported.

We therefore investigated pathology-related proteins and candidate biomarkers for therapeutic targets and disease assessments using a deep proteome called "next-generation proteomics," from sJIA sera of different disease activities.

\section{Results}

\section{Patients' characteristics}

We collected serum samples from 9 patients with SJIA (5-17 years old). As the study targeted a rare disease in children and its purpose was to search for differentially expressed proteins in the different disease phase and clarify the pathogenesis of the disease, we determined that the number of patients collected was sufficient for detailed proteomic analysis and assessing the quantitative changes in proteins.

The patients' characteristics and laboratory data at the time of serum collection are shown in Table 1. Sera were collected at different disease phases (MAS, active, and inactive phases) from seven of nine patients to compare the proteomes at different disease phases. Thus, 11 active-phase (including 6 MASphase) and 13 inactive-phase samples were used for the analysis. Some patients had few or mild joint symptoms at the onset of SJIA, and their arthritis was confirmed later. Thus, they did not have arthritis at the time of serum collection. The data collected from each patient did not differ substantially from the previously reported information. Therefore, the grouping of the data by disease phase was deemed reasonable. The age was consistent with the general age of SJIA patients, and the ferritin and IL-18 levels tended to be higher in patients with a high disease activity. Several patients were treated with biologics (e.g. tocilizumab) during the active phase.

\section{Identifying differentially expressed proteins during the active phase of sJIA and their characteristics}

Data analyses and differentially expressed proteins were performed using the workflow in Figure 1. Our MS analysis identified 2,727 proteins in the initial measurement of whole samples. According to our evaluation criteria, a total of 1,856 proteins were used in the analysis. We analyzed the changes in protein expression during the active phase by selecting five active-phase and eight-inactive phase samples from nine patients to avoid duplicating the same disease stage. The protein variation between the two groups is shown in Figure 2a. Among these, 607 proteins (32.7\%) were significantly expressed. A total of 566 proteins were upregulated ( $\geq 2$-fold) and 41 proteins were downregulated ( $\leq 0.5$-fold) (Figure $2 b$ ). These 
upregulated proteins included many acute-phase proteins with inflammatory activity, such as CRP, ferritin (ferritin light chain, heavy chain), serum amyloid A proteins (SAA-1, SAA-2), and heat shock proteins. We also detected proteins, such as soluble CD163 and IL-18, that had previously been reported to be elevated during the active phase and in cases with MAS complications (8-11). Among these proteins, the quantitative values of the MS analysis of CRP, ferritin, and IL-18 showed a high correlation with the laboratory data (IL-18 was measured by ELISA) (Supplemental figure 1). Based on these findings, our proteomic analysis accurately demonstrated the differences in protein expression in serum associated with the active phase of SJIA.

We also selected proteins from this group with levels that varied by disease activity. Using samples from the MAS group ( $n=6)$, active group $(n=5)$, and inactive group $(n=13)$, we performed an ANOVA and selected 591 proteins that were significantly differentially expressed. Then, we performed a principal component analysis (PCA) of these 591 proteins. The leading principal component (PC1) explained 77\% of the variance in the proteome data and consisted of 68 proteins (Figure $3 a$ and Supplemental table 1). All 68 PC1 proteins were differentially upregulated with levels that varied by disease activity (Figure 3b). We also labeled these proteins URPs (upregulated proteins) and deemed them potentially reflective of disease activity. These URPs and their functional characteristics were investigated, as explained in the following section.

\section{The functional enrichment and pathway analysis of URPs}

We performed a functional enrichment and pathway analysis to characterize the URPs. The majority of URPs were cytosolic proteins (GO:0005829). Classification by biological process revealed many GO terms that were related to immunity, e.g. immune-effector processes (GO:0002252), neutrophil activation (G0:0042119), neutrophil degranulation (G0:0043312), and chemokine production (G0:0032602) (Supplemental figure 2). Notably, the top enriched KEGG pathways for URPs included Proteasome (hsa03050). Identified proteasome subunits showed high expression in the active phase, suggesting that proteasome might play a role in the sJIA pathogenesis.

Next, we performed a protein-protein interaction analysis of URPs using STRING. For this analysis, we included identified downregulated proteins to further understand the pathogenesis. The major functional groups of these proteins are shown in Figure 4. These proteins, including proteasome subunits, had strong interactions with each other. Interestingly, some downregulated proteins that constitute growth factor binding (GO:0019838) and the PI3K-Akt signaling pathway (hsa04151) were revealed to be involved in URPs.

\section{Candidate biomarkers}

We selected four proteins from URPs; leucine aminopeptidase 3 (LAP3), guanylate-binding protein 1 (GBP1), heme oxygenase 1 (HMOX1), and bone morphogenetic protein 10 (BMP10), as candidate biomarkers based on their differential expression with high fold changes depending on the disease activity phase and/or as core proteins in the network analysis. 
A comparison of the quantitative expression values and the fold change of the four proteins (LAP3, GBP1, HMOX1, and BMP10) is shown in Table 2. These proteins, as well as the conventional biomarkers of inflammatory proteins (CRP, ferritin, and SAA), showed high quantitative values in the MS analysis and their expression varied depending on the disease stage. Furthermore, these proteins were highly correlated with each other (Figure 5a).

We also assessed the time-course expression of four proteins and conventional biomarkers in a patient with repeated relapses. This patient suffered from repeated complications of MAS despite receiving various immunosuppressive therapies (e.g. methylprednisolone pulse therapy, dexamethasone palmitate, cyclosporine, and etoposide). The clinical course is shown in Figure 5b. Biomarker candidate proteins were increased similar to conventional biomarkers, suggesting that these proteins may be useful for monitoring the SJIA disease activity.

\section{Discussion}

This study investigated differentially expressed serum proteins associated with sJIA disease activity using next-generation proteomics. The main finding was the identification of 68 URPs with elevated levels during the active phase among the 2,727 proteins obtained from MS. These URPs included proteasomerelated proteins and biomarker candidate proteins, such as LAP3, whose expression varies drastically by active phase; these proteins might be associated with the pathology.

As few specific biomarkers that can indicate and evaluate the sJIA disease activity have been identified, discovering novel biomarkers is very important in clinical practice. We identified proteins that were upregulated in active sJIA and might be useful for predicting relapse and making treatment decisions. In addition, the identification of proteins with a constitutively differential expression might facilitate our understanding of the underlying pathogenesis of sJIA.

The biomarker candidate proteins (LAP3, GBP1, HMOX1, and BMP10) that we selected were detected with high levels among URPs, and their expression levels varied depending on the disease stage. Since these proteins can be measured with high reproducibility using MS, they have the potential to be useful as serum biomarkers for a wide range of clinical applications in the future. Although further studies are needed, these proteins are also located in the inflammatory pathway for disease activity of sJIA and might be useful for the differential diagnosis.

We also successfully identified unprecedented numbers of serum proteins that proved useful for a functional and pathway analysis through careful preparation and next-generation proteomics technology. As serum protein concentrations are known to have a wide, dynamic range, most candidate biomarker proteins are low in abundance and cannot be detected by conventional preparation methods due to the influence of a high abundance of serum components, such as albumin(14). These techniques may be used to investigate serum biomarkers of other diseases in the future. 
LAP3 was the most highly expressed of the candidate biomarker proteins. This protein is not only involved in intracellular protein processing and regular turnover but is also assumed to be associated with inflammation via neutrophil activation and degranulation $(15,16)$. The dysregulation of LAP3 expression is also thought to cause changes in cell proliferation, invasion, and angiogenesis by altering peptide activation and has been reported as a candidate biomarker for the early diagnosis and therapeutic evaluation of colorectal and breast cancer(17). Interestingly, LAP3 and GBP1 were identified as proteins strongly upregulated by type 1 interferons (IFNs), along with CXCL9 and 10 and CCL2 and 8(18). CXCL9 is also upregulated during the MAS phase(19), indicating its involvement in the activation of inflammation along with IFN- $y$. Heme oxygenase (HMOX) is a potent anti-inflammatory and antioxidant rate-limiting enzyme that is involved in the degradation of heme to biliverdin, free ion, and carbon monoxide (CO) $(20,21)$. HMOX-1 is distributed in the liver, spleen, and endothelium with rapid induction in the presence of stressors. Recently, some reports have shown that HMOX1 deficiency exhibits hyperinflammation and features that are similar to MAS (22); thus, HMOX1 is considered an important protein in the regulation of systemic inflammation. In addition, it has been reported that HMOX1 levels are increased in patients with sJIA and adult Still's disease $(12,21,23)$. In this study, the network analysis showed that HMOX1 is a protein involved in inflammatory pathways and quantitative measurement by MS revealed that it was highly expressed. The expression change of BMP10 between MAS and the active phase was the most variable among the identified proteins. BMP10 is a circulating cytokine with an important role in endothelial homeostasis, and high BMP10 levels may increase the recruitment of monocytes to the vascular endothelium(24). This protein might be a useful biomarker for the early detection of complications and disease progression.

Important proteins to consider when discussing the underlying pathogenesis of SJIA are proteasome subunits. Proteasomes are protein complexes that function as a ubiquitin-proteasome system and are involved in not only breaking down dead proteins but also many other essential cellular processes, such as cell cycle regulation and proliferation(25). Proteasomes are located in not only the cytoplasm but also the serum as circulating proteasomes (c-proteasomes). Although the exact function of c-proteasomes remains unclear, the c-proteasome levels are increased in various diseases, such as autoimmune diseases, malignancies, and sepsis(26, 27). Furthermore, in this analysis, in cases with repeated relapses, proteasomes levels were higher in the active phase as well as inactive phase than in the active phase of cases without relapse. Although further confirmation will be needed because of the small number of cases reviewed here, our result suggests that increased c-proteasomes in SJIA might not be located downstream of systemic inflammation but rather upstream of the pathogenesis of sJIA. On the other hand, the role of the functions constituted by the downregulated proteins revealed in this study is also unclear. Thus, further studies will be needed to clarify the role of c-proteasomes in the pathogenesis of SJIA, including in relation to the function of the downregulated proteins.

Two strengths of this study warrant mention. First, the sample collection was devised to ensure an effective biomarker search. The clinical course, symptoms, and treatment were carefully followed at the same institution, and samples were collected and analyzed. We were thus able to reduce biases, such as environmental and medical equipment factors that might affect the serum proteome analysis results. 
Second, as mentioned above, we performed careful serum preparation and proteome analyses using the latest technology, which allowed us to obtain a large amount of information from the serum. We detected new biomarker candidates and pathological conditions by analyzing them in combination with the clinical course.

However, several limitations of the present study also warrant mention. First, the number of patients was small, so some biomarkers we found may have been affected by the patients' characteristics. Further studies evaluating the biomarkers in a large number of patients are needed to confirm their usefulness. Another methodological limitation is that low-abundance proteins (such as cytokines and chemokines) cannot be identified in serum, even using deep proteome analyses. Further technological innovation in proteome analyses will be needed to identify new biomarkers.

\section{Conclusion}

In conclusion, we successfully identified a protein group that was differentially expressed during the active phase and analyzed its functions and pathways in SJIA by next-generation proteomics. In particular, some proteins such as LAP3, whose levels increase dynamically during the active phase, are expected to be useful for clinical applications, such as making diagnoses and performing therapeutic evaluations, in combination with previously reported biomarkers. The proteins, such as proteasomes, identified here might also provide clues to the pathogenesis of the disease.

\section{Materials And Methods}

\section{Study design}

We performed a cross-sectional study of patients treated for sJIA. The study was performed according to the Declaration of Helsinki principles and approved by the ethics review board of Chiba Children's Hospital, Chiba, Japan. (Approval number, 2020-022). Written informed consent was obtained from the study participants and/or their guardians.

\section{Setting and participants}

We recruited patients with SJIA at Chiba Children's Hospital in October 2020. Chiba Children's Hospital serves most pediatric patients with rheumatic disease in the area due to the small number of centers treating pediatric rheumatic diseases. The eligible participants were patients who had previously been diagnosed with SJIA based on the International League of Associations for Rheumatology (ILAR) classification criteria(28) and had undergone treatment at Chiba Children's Hospital between April 2013 and March 2020. The exclusion criteria were a lack of medical records, complications of other rheumatic diseases, complications of acute infection at time of serum collection, or other conditions that might induce inflammation, such as surgery, injury, and malignancy. 
The patient's clinical symptoms and laboratory findings were obtained retrospectively from their medical records. The diagnosis of MAS was based on the 2016 EULAR/ACR/PRINTO classification criteria(29). The criteria for active-phase sJIA were typical symptoms, such as a fever, arthritis, hepatosplenomegaly, rash, and generalized lymphadenopathy, and increased CRP levels $(>0.3 \mathrm{mg} / \mathrm{dL})$.

\section{Sample preparation for proteome analyses}

Highly abundant serum proteins were depleted from $10 \mu$ of serum using a High Select Top 14 Abundant Protein Depletion Mini Spin Column (Thermo Fisher Scientific, Waltham, MA, USA) according to the manufacturer's instructions. Depleted serum $(50 \mu \mathrm{l})$ was diluted with $150 \mu \mathrm{l}$ of $100 \mathrm{mM}$ Tris-HCl pH 8.5 and $2 \%$ sodium dodecyl sulfate (SDS) and treated with $10 \mathrm{mM}$ dithiothreitol at $50{ }^{\circ} \mathrm{C}$ for $30 \mathrm{~min}$. The sample was then alkylated with $30 \mathrm{mM}$ iodoacetamide in the dark at room temperature for $30 \mathrm{~min}$ and subjected to cleanup and digestion with single-pot solid phase-enhanced sample preparation (SP3)(30).

Two types of beads (hydrophilic and hydrophobic Sera-Mag Speed-Beads; Cytiva, Marlborough, MA, USA) were used for the SP3 method. These beads were combined at a 1:1 (v/v) ratio, rinsed with distilled water, and reconstituted in $500 \mathrm{mM}$ Tris- $\mathrm{HCl} \mathrm{pH} 7.0$ at $10 \mu \mathrm{g}$ solids $/ \mu \mathrm{l}$. The reconstituted beads $(20 \mu \mathrm{l})$ were then added to the alkylated sample followed by ethanol to bring the final concentration to $75 \%(\mathrm{v} / \mathrm{v})$, with mixing for $20 \mathrm{~min}$. The beads were subsequently immobilized on a magnetic rack. The supernatant was discarded, and the pellet was rinsed with $80 \%$ ethanol and $100 \%$ acetonitrile (ACN). The beads were then resuspended in $40 \mu \mathrm{l}$ of $50 \mathrm{mM}$ Tris-HCl pH 8.0 with $1 \mu \mathrm{g}$ trypsin/Lys-C Mix (Promega, Madison, WI, USA) and digested by gentle agitation at $37^{\circ} \mathrm{C}$ overnight. The digested sample was acidified with $150 \mu \mathrm{l}$ of $0.1 \%$ Trifluoroacetic Acid (TFA) and then desalted using GL-Tip SDB (GL Sciences Inc., Tokyo, Japan) according to the manufacturer's instructions, followed by drying with a centrifugal evaporator. The dried peptides were redissolved in 3\% ACN and $0.1 \%$ formic acid and transferred to a hydrophilic-coated lowadsorption vial (ProteoSave vial; AMR Inc., Tokyo, Japan).

\section{Proteome analyses}

Peptides were directly injected onto a $75 \mu \mathrm{m} \times 40 \mathrm{~cm}$ PicoFrit emitter (New Objective, Woburn, MA, USA) packed in-house with C18 core-shell particles (CAPCELL CORE MP $2.7 \mu \mathrm{m}, 160$ Å material; Osaka Soda Co., Ltd., Osaka, Japan) at $60^{\circ} \mathrm{C}$ and then separated with a 120 -min gradient at $100 \mathrm{nl} / \mathrm{min}$ using an UltiMate 3000 RSLCnano LC system (Thermo Fisher Scientific, Waltham, MA, USA). Peptides eluting from the column were analyzed on a Q Exactive HF-X (Thermo Fisher Scientific) for overlapping window dataindependent acquisition (DIA)-MS(13, 31). MS1 spectra were collected in the range of $495-745 \mathrm{~m} / \mathrm{z}$ at 30,000 resolution to set an automatic gain control target of $3 \times 10^{6}$ (unit) and maximum injection time of 55 (unit). MS2 spectra were collected at $>200 \mathrm{~m} / \mathrm{z}$ at 45,000 resolution to set an automatic gain control target of $3 \times 10^{6}$ (unit), maximum injection time of "auto," and stepped normalized collision energy of $22 \%$, $26 \%$, and $30 \%$. The isolation width for MS2 was set to $4 \mathrm{~m} / \mathrm{z}$, and overlapping window patterns of 500 $740 \mathrm{~m} / \mathrm{z}$ were used for window placements optimized by Skyline. 
The MS files were searched against a human spectral library using Scaffold DIA (Proteome Software, Inc., Portland, OR, USA). The human spectral library was generated from the human protein sequence database (UniProt id UP000005640, reviewed, canonical) established by Prosit. The Scaffold DIA search parameters were as follows: experimental data search enzyme, trypsin; maximum missed cleavage sites, 1; precursor mass tolerance, 8 ppm; fragment mass tolerance, 8 ppm; static modification, cysteine carbamidomethylation. The protein identification threshold was set at $<1 \%$ for both peptide and protein false discovery rates. The peptide quantification was calculated by the EncyclopeDIA algorithm(32) in Scaffold DIA. For each peptide, the four highest-quality fragment ions were selected for quantitation. The protein quantitative value was estimated from the summed peptide quantitative values. Total quantitative values were calculated by normalizing the protein quantitative values between samples.

\section{Enzyme-linked immunosorbent assays (ELISAs)}

Serum IL-6 and IL-18 levels were measured using a commercial ELISA (IL-18: MBL, Nagoya, Japan; IL-6: R\&D Systems, Inc., Minneapolis, MN, USA), according to the manufacturer's instructions.

\section{The statistical and bioinformatics analysis of the data}

We performed statistical analyses and generated hierarchical clustering and heatmaps using the JMP Pro13 software program (SAS Institute Inc., Cary, NC, USA) and Qlucore omics explorer software program (Qlucore AB, Lund, Sweden).

We used proteins with at least three peptide counts that were detected in at least one subject for the subsequent analysis, with the exclusion of proteins with missing values in some subjects. After conducting $\log _{2}$ transformation for the quantified continuous values, an independent sample two-sided $t$ test or an analysis of variance (ANOVA) was used. P values of $<0.05$, with a confidence interval of $95 \%$, were considered to indicate statistical significance.

We also used a principal component analysis in our study to reduce the identified proteins into a few dimensions that reduce a large amount of the variability of the original values. The first principal component (PC1) explains the highest amount of variability of the original data. Furthermore, correlations of the identified proteins were investigated using Spearman's correlation coefficient method.

An analysis of functional Gene Ontology (GO) terms and the Kyoto Encyclopedia of Genes and Genomes (KEGG) pathway were performed with the online tool Search Tool for the Retrieval of Interacting Genes (STRING v11; http://string-db.org/) (33). An association network of the identified proteins was also established using the STRING database with the highest confidence interaction score. The proteins that showed elevated serum values were referred to the Human Protein Atlas (HPA;

https://www.proteinatlas.org/) (34).

\section{Declarations}




\section{Acknowledgments}

\section{The authors truly value the patients and for their great participation.}

The authors above have no relevant financial disclosures or benefits from commercial sources that could create a potential conflict of interest. This work was funded by Ministry of Education, Culture, Sports, Science and Technology (MEXT) Grant-in-Aid for Scientific Research (C) (no. 18K08383 to YI).

\section{Author contributions statement}

HS and YI designed the study. HS, YI, DK, KM, TY, AY, MT and AH treated patients and also recruited them to the study. HS, YK, DN, RN performed the experimental work. HS, YI and YK analyzed and interpreted the data. HS and YI wrote the manuscript. 00 supervised the interpretation of the data. 00 and NS provided guidance and made important contributions to the writing of the manuscript. All authors read, made improvements and approved the final version.

Competing interests: None declared.

Provenance and peer review: Not commissioned; externally peer reviewed.

Data sharing statement: The authors have no objection and are willing to share the data used for the current research article.

\section{References}

1. Martini A, et al. Toward New Classification Criteria for Juvenile Idiopathic Arthritis: First Steps, Pediatric Rheumatology International Trials Organization International Consensus. $J$ Rheumatol.46(2),190-7 (2019).

2. Mellins ED, Macaubas C, Grom AA. Pathogenesis of systemic juvenile idiopathic arthritis: some answers, more questions. Nat Rev Rheumatol.7(7), 416-26 (2011);

3. Vastert SJ, Kuis W, Grom AA. Systemic JIA: new developments in the understanding of the pathophysiology and therapy. Best Pract Res Clin Rheumatol.23(5), 655-64 (2009).

4. Schulert GS, Grom AA. Macrophage activation syndrome and cytokine-directed therapies. Best Pract Res Clin Rheumatol. 28(2), 277-92 (2014).

5. Schulert GS, et al. Effect of Biologic Therapy on Clinical and Laboratory Features of Macrophage Activation Syndrome Associated With Systemic Juvenile Idiopathic Arthritis. Arthritis Care Res (Hoboken).70(3), 409-19 (2018).

6. Mizuta M, et al. Serum ferritin levels as a useful diagnostic marker for the distinction of systemic juvenile idiopathic arthritis and Kawasaki disease. Mod Rheumatol.26(6), 929-32 (2016).

7. Eloseily EMA, Minoia F, Crayne CB, Beukelman T, Ravelli A, Cron RQ. Ferritin to Erythrocyte Sedimentation Rate Ratio: Simple Measure to Identify Macrophage Activation Syndrome in Systemic 
Juvenile Idiopathic Arthritis. ACR Open Rheumatol.1(6), 345-9 (2019).

8. Shimizu M, et al. Distinct cytokine profiles of systemic-onset juvenile idiopathic arthritis-associated macrophage activation syndrome with particular emphasis on the role of interleukin-18 in its pathogenesis. Rheumatology (Oxford).49(9), 1645-53 (2010).

9. Takahara T, Shimizu M, Nakagishi Y, Kinjo N, Yachie A. Serum IL-18 as a potential specific marker for differentiating systemic juvenile idiopathic arthritis from incomplete Kawasaki disease. Rheumatol Int.35(1), 81-4 (2015).

10. Kudela H, Drynda S, Lux A, Horneff G, Kekow J. Comparative study of Interleukin-18 (IL-18) serum levels in adult onset Still's disease (AOSD) and systemic onset juvenile idiopathic arthritis (SJIA) and its use as a biomarker for diagnosis and evaluation of disease activity. BMC Rheumatol.3,4; 10.1186/s41927-019-0053-z (2019).

11. Sakumura N, et al. Soluble CD163, a unique biomarker to evaluate the disease activity, exhibits macrophage activation in systemic juvenile idiopathic arthritis. Cytokine. 110,459-65 (2018).

12. Shimizu M, Yachie A. Compensated inflammation in systemic juvenile idiopathic arthritis: role of alternatively activated macrophages. Cytokine.60(1), 226-32 (2012).

13. Kawashima Y, et al. Optimization of Data-Independent Acquisition Mass Spectrometry for Deep and Highly Sensitive Proteomic Analysis. Int J Mol Sci.20(23); 10.3390/ijms20235932 (2019).

14. Anderson NL, Anderson NG. The human plasma proteome: history, character, and diagnostic prospects. Mol Cell Proteomics.1(11), 845-67 (2002).

15. Huang $\mathrm{H}$, Tanaka $\mathrm{H}$, Hammock BD, Morisseau $\mathrm{C}$. Novel and highly sensitive fluorescent assay for leucine aminopeptidases. Anal Biochem.391(1), 11-6 (2009).

16. Didangelos A. COVID-19 Hyperinflammation: What about Neutrophils? mSphere.5(3); 10.1128/mSphere.00367-20 (2020)

17. Q Y, MH R, JY. W. Proteomic profiling of antibody-inducing immunogens in tumor tissue identifies PSMA1, LAP3, ANXA3, and maspin as colon cancer markers. Onco Targets Ther.9, 3996-4019 (2018).

18. Forster S. Interferon signatures in immune disorders and disease. Immunol Cell Biol.90(5), 520-7 (2012).

19. Mizuta M, Shimizu M, Inoue N, Nakagishi Y, Yachie A. Clinical significance of serum CXCL9 levels as a biomarker for systemic juvenile idiopathic arthritis associated macrophage activation syndrome. Cytokine.119, 182-7 (2019).

20. Miyazaki T, et al. Serum HO-1 is useful to make differential diagnosis of secondary hemophagocytic syndrome from other similar hematological conditions. Int J Hematol.91(2), 229-37 (2010).

21. Takahashi A, et al. The role of heme oxygenase- 1 in systemic-onset juvenile idiopathic arthritis. Mod Rheumatol.19(3), 302-8 (2009).

22. Chau AS, et al. Heme oxygenase-1 deficiency presenting with interstitial lung disease and hemophagocytic flares. Pediatr Rheumatol Online J.18(1), 80; 10.1186/s12969-020-00474-1 (2020). 
23. Kirino Y, et al. Increased serum HO-1 in hemophagocytic syndrome and adult-onset Still's disease: use in the differential diagnosis of hyperferritinemia. Arthritis Res Ther.7(3), R616-24 (2005).

24. Mitrofan CG, et al. Bone morphogenetic protein 9 (BMP9) and BMP10 enhance tumor necrosis factor-alpha-induced monocyte recruitment to the vascular endothelium mainly via activin receptorlike kinase 2. J Biol Chem.292(33), 13714-26 (2017).

25. Budenholzer L, Cheng CL, Li Y, Hochstrasser M. Proteasome Structure and Assembly. J Mol Biol.429(22), 3500-24 (2017).

26. Sixt SU, Dahlmann B. Extracellular, circulating proteasomes and ubiquitin - incidence and relevance. Biochim Biophys Acta.1782(12), 817-23 (2008).

27. Ramdas P, Radhakrishnan AK, Abdu Sani AA, Kumari M, Anandha Rao JS, Abdul-Rahman PS. Advancing the Role of Gamma-Tocotrienol as Proteasomes Inhibitor: A Quantitative Proteomic Analysis of MDA-MB-231 Human Breast Cancer Cells. Biomolecules. 10(1); 10.3390/biom10010019 (2019).

28. Petty RE, et al. International League of Associations for Rheumatology classification of juvenile idiopathic arthritis: second revision, Edmonton, 2001. J Rheumatol. 31(2), 390-2 (2004).

29. Ravelli A, et al. 2016 Classification Criteria for Macrophage Activation Syndrome Complicating Systemic Juvenile Idiopathic Arthritis: A European League Against Rheumatism/American College of Rheumatology/Paediatric Rheumatology International Trials Organisation Collaborative Initiative. Arthritis Rheumatol.68(3), 566-76 (2016).

30. Sielaff M, et al. Evaluation of FASP, SP3, and iST Protocols for Proteomic Sample Preparation in the Low Microgram Range. J Proteome Res. 16(11), 4060-72 (2017).

31. Amodei D, et al. Improving Precursor Selectivity in Data-Independent Acquisition Using Overlapping Windows. J Am Soc Mass Spectrom.30(4), 669-84 (2019).

32. Searle BC, et al. Chromatogram libraries improve peptide detection and quantification by data independent acquisition mass spectrometry. Nat Commun. 9(1),5128; 10.1038/s41467-018-07454-w (2018).

33. Szklarczyk D, et al. STRING v11: protein-protein association networks with increased coverage, supporting functional discovery in genome-wide experimental datasets. Nucleic Acids Res.47(D1), D607-D13 (2019).

34. Thul PJ, Lindskog C. The human protein atlas: A spatial map of the human proteome. Protein Sci.27(1), 233-44 (2018).

\section{Tables}

\section{Table 1. Clinical characteristics of the participants}

MAS, active, and inactive are defined in the Methods.

IQR, interquartile range; MAS, macrophage activation syndrome; PSL, prednisolone. 


\begin{tabular}{|c|c|c|c|c|}
\hline Characteristics & Total $(n=24)$ & MAS $(n=6)$ & Active $(n=5)$ & $\begin{array}{l}\text { Inactive } \\
(n=13)\end{array}$ \\
\hline $\begin{array}{l}\text { Age at the time of } \\
\text { serum collection } \\
\text { (years; median/IQR) }\end{array}$ & $5(4-12)$ & $4(3.5-6)$ & $4(3.5-7)$ & $9(4-14.3)$ \\
\hline Sex (male/female) & $3 / 21$ & $0 / 6$ & $0 / 5$ & $3 / 11$ \\
\hline \multicolumn{5}{|l|}{$\begin{array}{l}\text { Laboratory findings } \\
\text { (median/IQR) }\end{array}$} \\
\hline White blood cells count $(/ \mu \mathrm{L})$ & $\begin{array}{l}7,300 \\
(4,500- \\
15,800)\end{array}$ & $\begin{array}{l}3,750 \\
(1,125-2,760)\end{array}$ & $\begin{array}{l}20,300 \\
(11,500- \\
26,900)\end{array}$ & $\begin{array}{l}6,650 \\
(4,650- \\
10,475)\end{array}$ \\
\hline Hemoglobin (g/dL) & $\begin{array}{l}11.9 \\
(10.4-13.0)\end{array}$ & $\begin{array}{l}10.2 \\
(9.0-11.4)\end{array}$ & $\begin{array}{l}10.5 \\
(9.7-14.3)\end{array}$ & $\begin{array}{l}12.5 \\
(11.8-13.3)\end{array}$ \\
\hline Platelet counts $\left(\times 10^{4} / \mathrm{dL}\right)$ & $\begin{array}{l}24.2 \\
(15.2-38.0)\end{array}$ & $\begin{array}{l}11.9 \\
(6.3-28.8)\end{array}$ & $\begin{array}{l}29.5 \\
(15.0-51.5)\end{array}$ & $\begin{array}{l}25.4 \\
(21.5-39.4)\end{array}$ \\
\hline C-reactive protein $(\mathrm{mg} / \mathrm{dL})$ & $\begin{array}{l}0.12 \\
(0.04-1.78)\end{array}$ & $\begin{array}{l}2.98 \\
(1.5-7.5)\end{array}$ & $\begin{array}{l}0.41 \\
(0.2-10.7)\end{array}$ & $\begin{array}{l}0.06 \\
(0.04-0.1)\end{array}$ \\
\hline Ferritin $(\mathrm{ng} / \mathrm{mL})$ & $\begin{array}{l}164 \\
\left(33.3^{-}\right. \\
2746.6)\end{array}$ & $\begin{array}{l}7,425 \\
(2,688- \\
24,156)\end{array}$ & $\begin{array}{l}1,860 \\
(164-2,506)\end{array}$ & $\begin{array}{l}43 \\
(18.5-123)\end{array}$ \\
\hline Interleukin-18 (pg/ml) & $\begin{array}{r}14,375 \\
(5,168- \\
30,644)\end{array}$ & $\begin{array}{l}15,574 \\
(10,954- \\
27,610)\end{array}$ & $\begin{array}{l}98,415 \\
(33,678- \\
125,000)\end{array}$ & $\begin{array}{l}7,672 \\
(2,477- \\
14,375)\end{array}$ \\
\hline $\begin{array}{l}\text { Aspartate aminotransferase } \\
(\mathrm{IU} / \mathrm{L})\end{array}$ & $\begin{array}{l}34.0 \\
(20.0-80.7)\end{array}$ & $\begin{array}{l}136.5 \\
(87.6-236.8)\end{array}$ & $\begin{array}{l}63.0 \\
(29-80.5)\end{array}$ & $\begin{array}{l}22.5 \\
(18.8-31.8)\end{array}$ \\
\hline Fibrinogen (mg/dL) & $\begin{array}{l}261 \\
(183.5-365)\end{array}$ & $\begin{array}{l}303.5 \\
(240.3-402.0)\end{array}$ & $\begin{array}{l}323 \\
(189-649.8)\end{array}$ & $\begin{array}{l}189 \\
(158-192)\end{array}$ \\
\hline Triglyceride (mg/dL) & $\begin{array}{l}160 \\
(115.5-230)\end{array}$ & $\begin{array}{l}180.5 \\
(142.5-252.5)\end{array}$ & $\begin{array}{l}103 \\
(92.3-146)\end{array}$ & $\begin{array}{l}165 \\
(118-302)\end{array}$ \\
\hline \multicolumn{5}{|l|}{ Clinical symptoms (n, \%) } \\
\hline Fever & $7(28)$ & $6(100)$ & $1(20)$ & $0(0)$ \\
\hline Rash & $9(36)$ & $6(100)$ & $3(60)$ & $0(0)$ \\
\hline
\end{tabular}




\begin{tabular}{|lllll|}
$\begin{array}{l}\text { Hepatomegaly and/or } \\
\text { Splenomegaly }\end{array}$ & $6(24)$ & $4(67)$ & $0(0)$ & $2(28)$ \\
\hline Arthritis & $4(16)$ & $1(16)$ & $3(60)$ & $0(0)$ \\
\hline Serositis & $1(4)$ & $1(16)$ & $0(0)$ & $0(0)$ \\
\hline Treatment $(n, \%)$ & & & & \\
\hline PSL & $23(92)$ & $5(83)$ & $5(100)$ & $13(93)$ \\
\hline Biologics & $10(40)$ & $0(0)$ & $2(40)$ & $8(57)$ \\
\hline
\end{tabular}

Table 2. Quantitative values and fold change by disease phase for candidate biomarkers and previous biomarkers.

Gene names registered in the human protein sequence database (UniProt id UP000005640, reviewed, canonical) are shown.

MAS, macrophage activation syndrome; LAP3, aminopeptidase 3; GBP1, guanylate-binding protein 1; HMOX1, heme oxygenase 1; BMP10, bone morphogenetic protein 10; CRP, C-reactive protein; FTH, ferritin heavy chain; SAA1, serum amyloid A-1 protein;

* $\mathrm{X} / \mathrm{Y}$ in fold change is the mean quantitative value of $\mathrm{X}$ divided by that of $\mathrm{Y}$. 


\begin{tabular}{|c|c|c|c|c|c|c|}
\hline & \multirow[t]{2}{*}{$\begin{array}{l}\text { Peptide } \\
\text { count }\end{array}$} & \multicolumn{3}{|c|}{$\begin{array}{l}\text { Average quantitative value } \\
\text { ( } \log _{2} \text { transformed) }\end{array}$} & \multicolumn{2}{|l|}{ Fold change } \\
\hline & & MAS & $\begin{array}{l}\text { Active } \\
\text { phase }\end{array}$ & $\begin{array}{l}\text { Inactive } \\
\text { phase }\end{array}$ & MAS/active* & Active/inactive ${ }^{\star}$ \\
\hline \multicolumn{7}{|c|}{$\begin{array}{l}\text { Candidate } \\
\text { biomarkers }\end{array}$} \\
\hline LAP3 & 25 & 28.696 & 26.627 & 22.069 & 4.2 & 23.6 \\
\hline GBP1 & 12 & 22.033 & 20.377 & 18.037 & 3.2 & 5.6 \\
\hline HMOX1 & 8 & 24.595 & 24.858 & 20.698 & 0.8 & 17.9 \\
\hline BMP10 & 5 & 25.366 & 20.423 & 20.309 & 30.7 & 1.1 \\
\hline \multicolumn{7}{|c|}{$\begin{array}{l}\text { Previous } \\
\text { biomarkers }\end{array}$} \\
\hline SAA2 & 14 & 28.253 & 24.631 & 19.130 & 12.3 & 45.3 \\
\hline CRP & 10 & 32.219 & 30.311 & 24.509 & 3.75 & 55.8 \\
\hline FTH & 6 & 25.157 & 21.612 & 17.775 & 11.7 & 14.3 \\
\hline
\end{tabular}

\section{Figures}




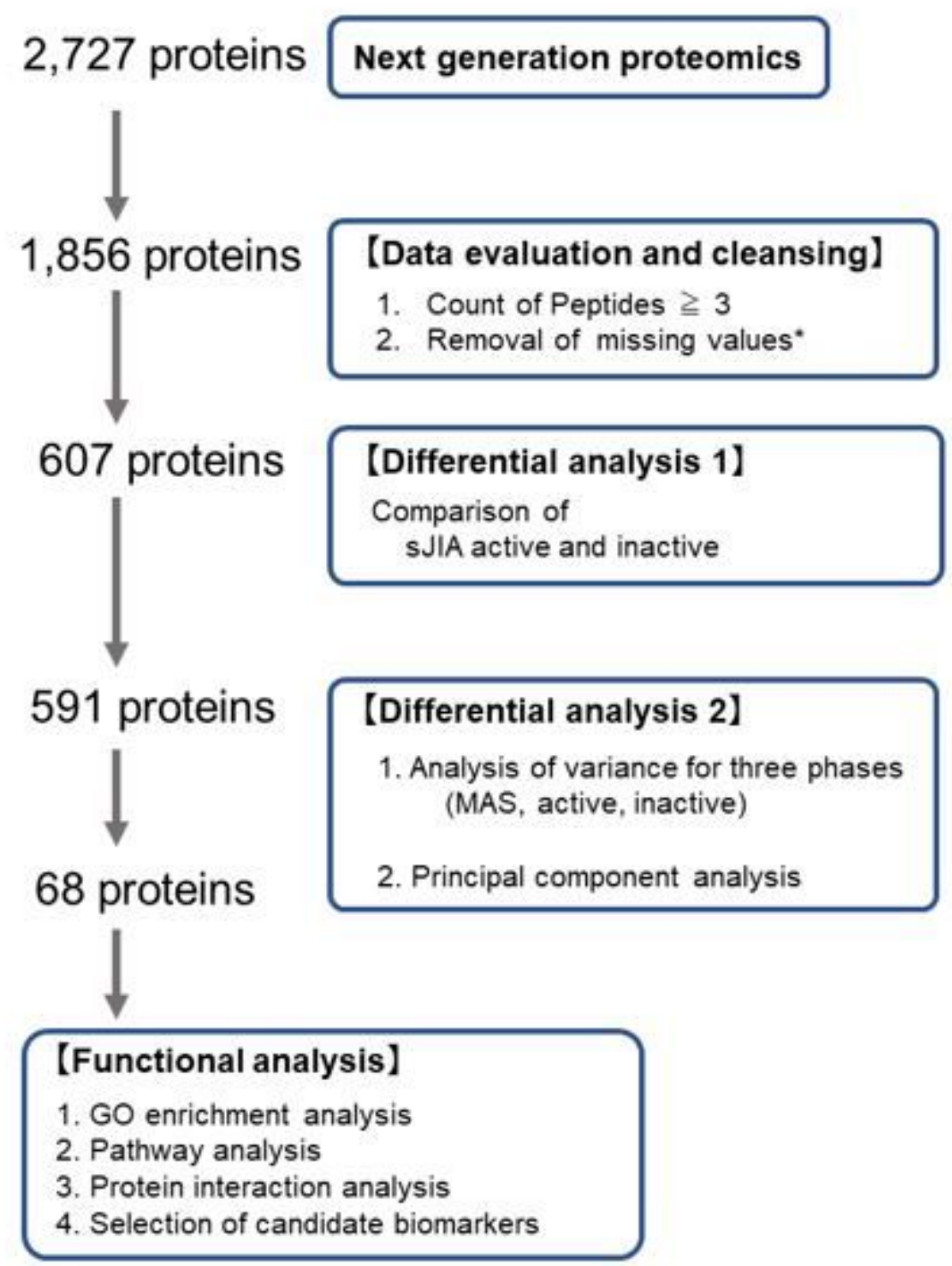

Figure 1

The analysis workflow. *We removed proteins that contained samples that could not be quantified as a missing value. SJIA, systemic juvenile idiopathic arthritis; MAS, macrophage activation syndrome; GO, gene ontology. 

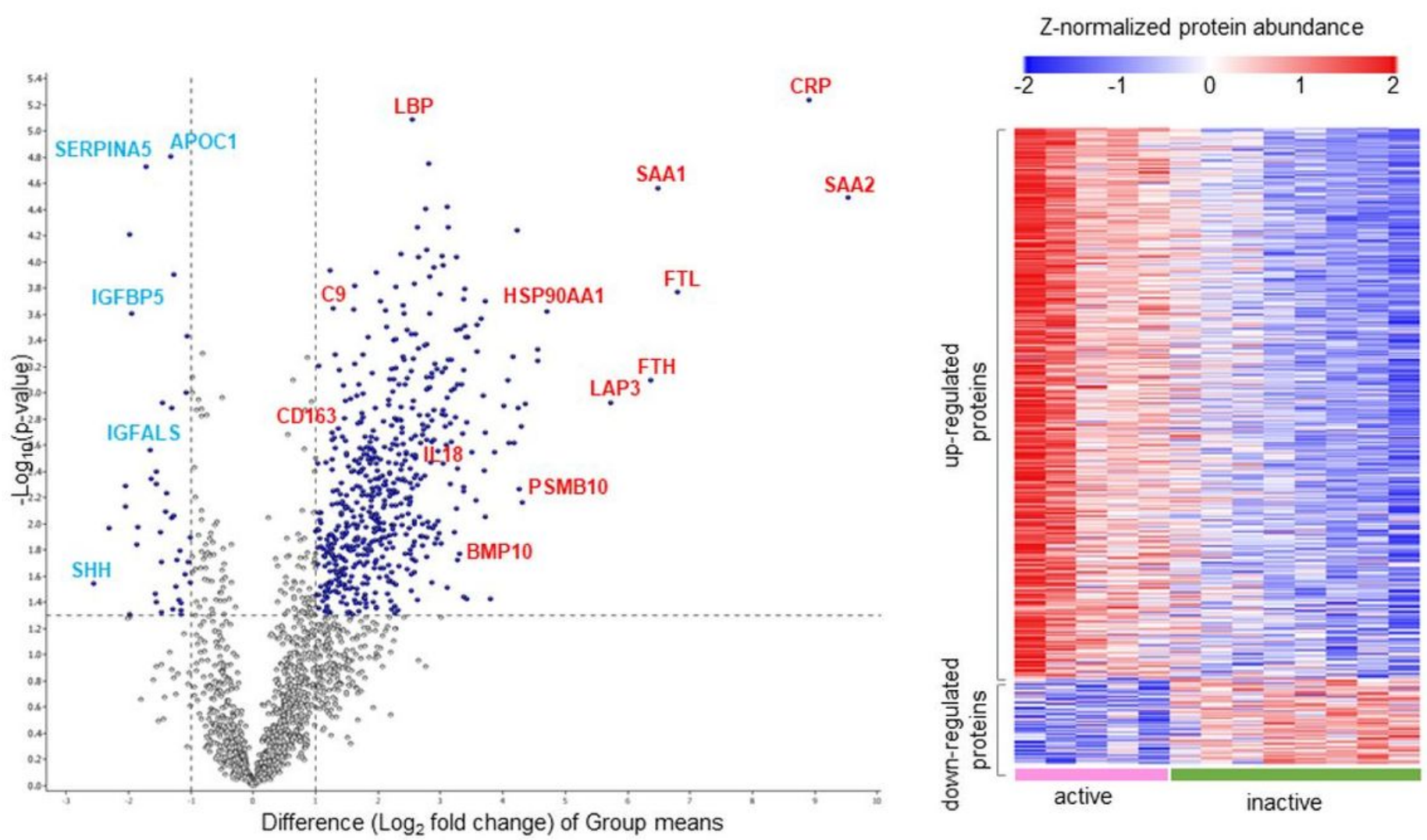

Figure 2

Proteins that are differently expressed in SJIA-active serum. (a) Volcano plot showing the expression in the active and inactive phases with fold changes and $p$-value. The dotted line on the horizontal axis represents $p=0.05$, and the dotted line on the vertical axis represents a 2 -fold change. (b) The heat map shows the protein groups that were significantly differentially expressed. The log-transformed protein quantitative values were standardized by z-score and color-coded. CRP, C-reactive protein; SAA1, serum amyloid A-1 protein; SAA2, serum amyloid A-2 protein; LBP, lipopolysaccharide-binding protein; $F T H$, ferritin heavy chain; FTL, ferritin light chain; HSP90AA1, Heat shock protein HSP 90-alpha; LAP3, aminopeptidase 3; IL18, interleukin-18; C9, complement component C9; BMP10, bone morphogenetic protein 10; PSMB10, proteasome subunit beta type-10; CD163, scavenger receptor cysteine-rich type 1 protein M130; APOC1, Apolipoprotein C-l; SERPINA5, Plasma serine protease inhibitor; IGFBP5, Insulin-like growth factor-binding protein 5; IGFALS, Insulin-like growth factor-binding protein complex acid labile subunit; $\mathrm{SHH}$, Sonic hedgehog protein. 


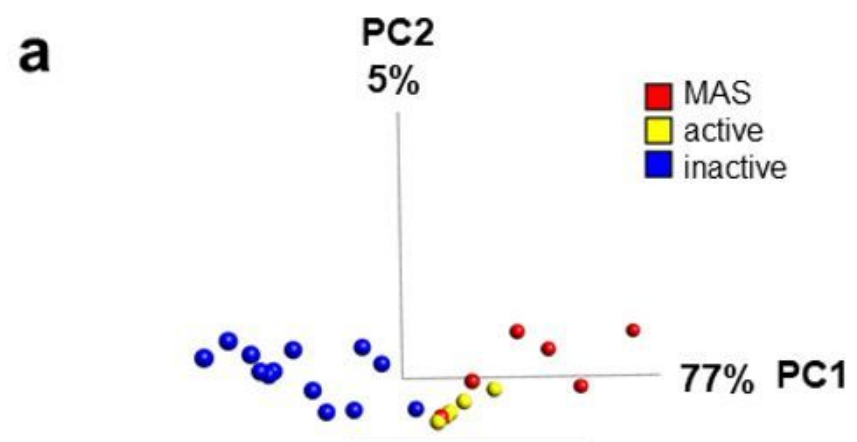

68 proteins

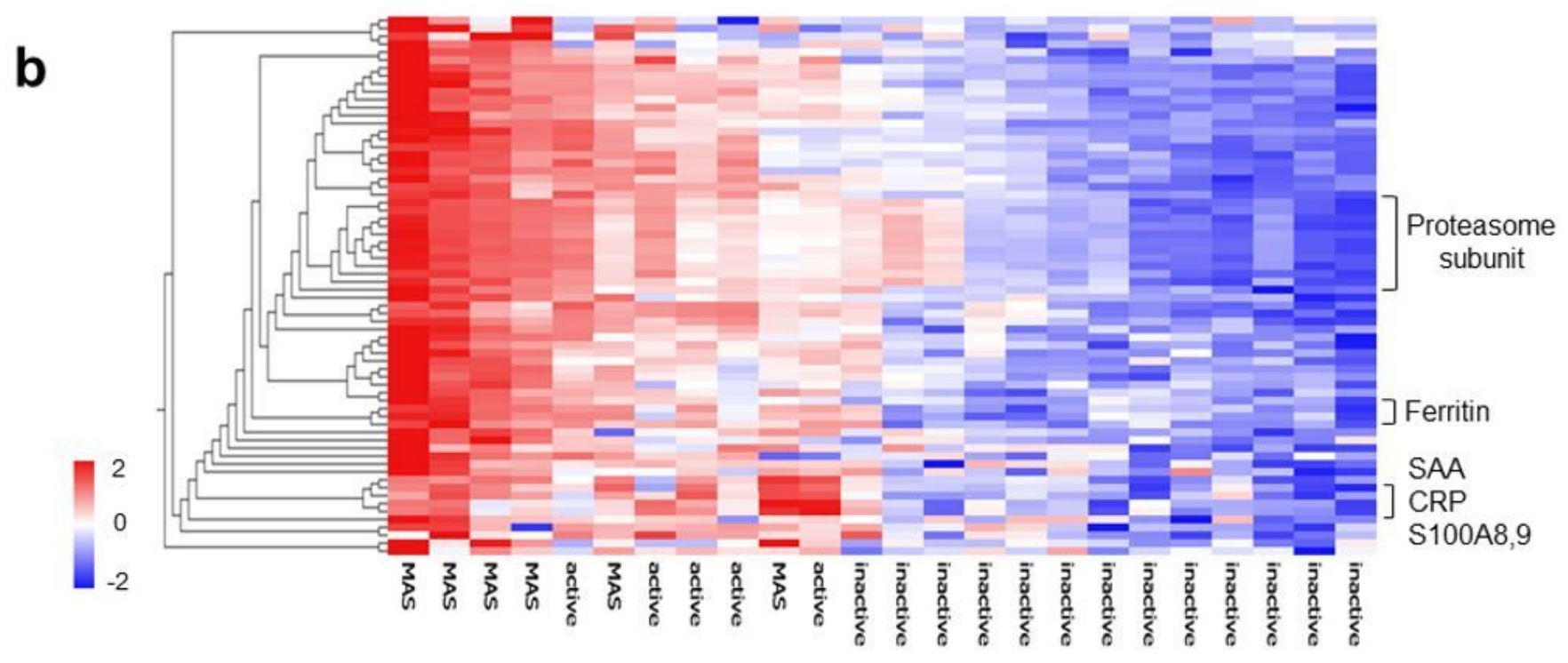

Figure 3

Proteins selected using PCA and a heat map and hierarchical cluster analysis of the proteins. (a) A principal component analysis and filtering of identified proteins were performed in conditions showing high PC1 values, which explains disease activity. (b) A heat map constructed of 68 proteins. The horizontal axis shows samples aligned by a PCA, and the vertical axis shows proteins that are listed in order to clustering analysis (See Supplemental table 1 for a list of the 68 selected proteins). PC, principal component; MAS, macrophage activation syndrome; CRP, C-reactive protein; SAA1, serum amyloid A-1 protein; S100A8,9, protein S100A-8, and 9. 


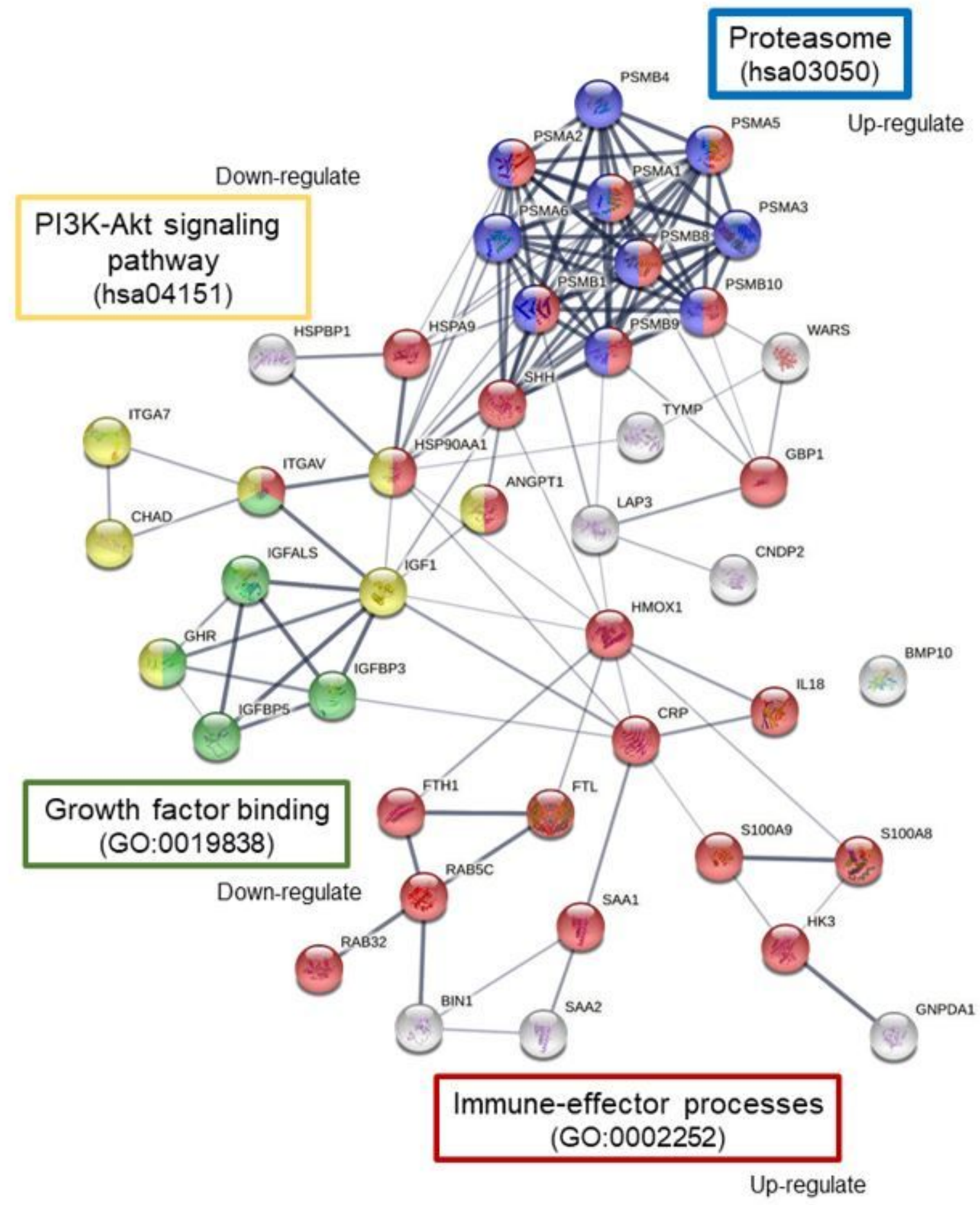

Figure 4

Results of a STRING-based interaction analysis of identified proteins. This schematic diagram represents the proteins and the functional groups of the proteins. Differentially expressed proteins were classified with the Gene Ontology and KEGG pathway, and essential proteins were selected. The thickness of the lines indicates the confidence level of the predicted interactions (confidence score $\geq 0.9$, thick lines; confidence score $\geq 0.4$, thin lines). Refer to Supplemental table 1 for the name of each protein gene. 
a

\begin{tabular}{|c|c|c|c|c|c|c|c|}
\hline CRP & & & & & & & \\
\hline 0.636 & IL-18 & & & & & & \\
\hline 0.775 & 0.572 & FTL & & & & & \\
\hline 0.668 & 0.492 & 0.967 & FTH & & & & \\
\hline 0.630 & 0.697 & 0.826 & 0.805 & LAP3 & & & \\
\hline $0.391^{*}$ & 0.479 & 0.700 & 0.703 & 0.836 & GBP1 & & \\
\hline 0.703 & 0.810 & 0.753 & 0.677 & 0.877 & 0.660 & HMOX1 & \\
\hline $0.474^{\circ}$ & $0.379^{\circ}$ & 0.605 & 0.618 & 0.688 & 0.648 & $0.488^{\circ}$ & BMP10 \\
\hline
\end{tabular}

b

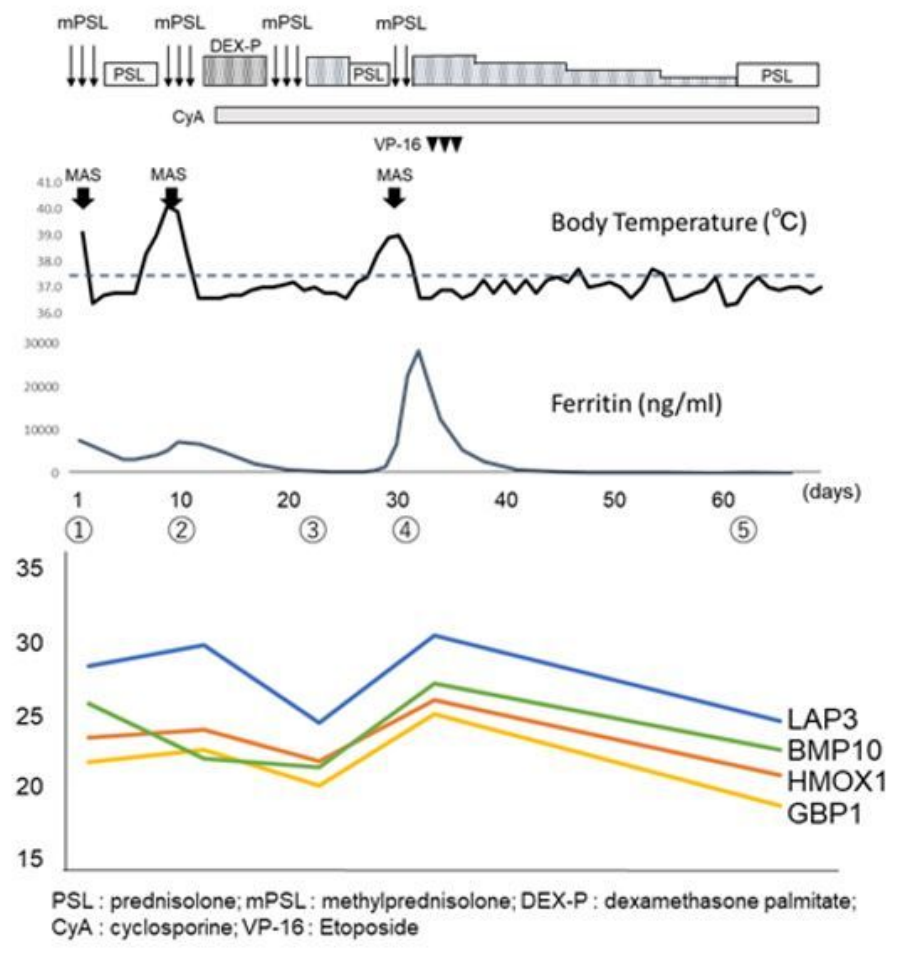

Figure 5

Correlation coefficients and the evaluation of candidate biomarkers. (a) The numbers indicate the correlation coefficient for each protein. Numbers with strong correlations $(>0.7)$ are shown in bold red type. Correlation coefficients that were not significantly different $(p \geq 0.05)$ are marked with an asterisk $(*)$. (b) Longitudinal course of a case with MAS. The details of therapeutic interventions are shown at the top. The lower area shows a line graph for candidate biomarkers. Serum was collected and analyzed at points (1) to (5). CRP, C-reactive protein; IL18, interleukin-18; FTL, ferritin light chain; FTH, ferritin heavy chain; LAP3, aminopeptidase 3; GBP1, guanylate-binding protein 1; HMOX1, heme oxygenase 1; BMP10, bone morphogenetic protein 10.

\section{Supplementary Files}

This is a list of supplementary files associated with this preprint. Click to download.

- supplementarymaterialSR.docx 